\title{
Excitation of Acoustic Waves in Plasmas
}

\author{
William A. Saxton \\ Gordon McKay Laboratory, Harvard University, Cambridge, Mass.
}

(Received October 13, 1964: revised November 16, 1964)

\begin{abstract}
This paper reports early results in an experimental study to determine the effects of perturbing weakly-ionized gaseous plasmas with acoustic waves emanating from transducers that operate in the audio and ultrasonic frequency ranges. Compact loudspeakers incorporated into cylindrical plasma discharge tubes provide the source of acoustic signals. Modulation of electromagnetic waves by the acoustically-disturbed plasma is measured in a unique rectangular cavity whose output is detected and fed to a wave analyzer. Resultant wave-analyzer responses indicate that the collision frequency is modulated in addition to the plasma frequency, and suggest that the variations in both are proportional to the magnitude of loudspeaker diaphragm deflection, as predicted by simple acoustic theory. Knowing that two forms of modulation occur simultaneously, a calibration scheme was developed to separate the variation $\Delta f_{p}$ in the plasma frequency from the total response. Measurements to date indicate that $\Delta N_{e} / N_{e}=\Delta N / N$ (where $N_{e}$ and $N$ are electron and neutral-molecule densities, respectively) for slightly-ionized gases which are subjected to low-frequency pressure variation in the order of $10^{-5}$ $\mathrm{mm} \mathrm{Hg}$, and that such variations produce plasma-frequency perturbations of $0-140 \mathrm{kc} / \mathrm{s}$ at plasma frequencies up to $800 \mathrm{Mc} / \mathrm{s}$.
\end{abstract}

\section{Introduction}

Although there has been a great deal of research on noise and oscillations in both laboratory and natural plasmas, the major emphasis has been on those fluctuations which are self-excited by the medium itself. Recently, however, experimentalists have turned their attention to new methods for intentionally perturbing a plasma, to studies of the resultant electron- or ion-density variations, and to the subsequent influence of such perturbations in the propagation of electromagnetic and electroacoustic waves [Goldstein, Roux, and Dayton, 1963; Alexeff and Jones, 1963; Kino and Allen, 1962]. This paper describes the first stage of one such investigation in which the perturbing devices are acoustic transducers operating in the audio and ultrasonic ranges.

Electrically neutral, slightly ionized isotropic gases, consisting of electrons, an equal number of ions per unit volume, and neutral molecules far in excess of electrons and ions, can be conveniently characterized by an electron plasma frequency

$$
f_{p}=\frac{e}{2 \pi} \sqrt{\frac{N_{e}}{m \epsilon_{0}}},
$$

( $e$ and $m$ are the electronic charge and mass, respec-) tively, $\epsilon_{0}$ is the permittivity of free space, $N_{e}$ is the electron density. ${ }^{1}$ The research reported in this paper was supported in part by the Office of Naval Re-
search under Contract Nonr-1866(26) and in part by the National Science Foundation under Grant GP 2242 and an electron collision frequency $\nu_{c}$-in collisions per second-which describes the directed momentum transfer between an electron and the other particles in the medium. In a simple model of such a gas, the properties of an electromagnetic wave passing through it depend upon $f_{p}$ and $\nu_{c}$ through the complex dielectric constant.

$$
\xi=\epsilon-\frac{j \sigma}{\omega}=\epsilon_{0}\left(1-\frac{\omega_{p}^{2}}{\nu_{c}^{2}+\omega^{2}}+j \frac{\omega_{p}^{2} \frac{\nu_{c}}{\omega}}{\nu_{c}^{2}+\omega^{2}}\right),
$$

where $\omega$ is the electromagnetic angular frequency, $\epsilon$ is the dielectric constant and $\sigma$ is the conductivity; $\omega_{p}=2 \pi f_{p}$. Therefore, any perturbation of $f_{p}$ and $\nu_{c}$ can, in principle, be detected by considering the plasma as a medium with varying dielectric constant and conductivity, and observing the modulation of an electromagnetic signal propagating through it.

Menzel [1964], in reviewing the various interactions in the ionosphere that take place because of nonlinear processes, pointed out that acoustic disturbances from rockets, missiles, explosions and collisions of solar ion clouds with the upper atmosphere are all potential sources of acoustic waves which can impress modulation on electromagnetic waves, and asked whether or not the various kinds of interaction which result could be studied experimentally. In addition, Drummond [1962] extended an ion-wave theory to include nonlinearities and showed it possible to propagate electromagnetic waves through acoustically disturbed plasmas at frequencies below the plasma frequency where a negative dielectric constant ordinarily forbids the propagation of RF fields. Although 
Sessler and Schroeder [1962] experimentally observed the interaction of noise generated by a d-c discharge with externally applied sound waves, their interest was exclusively in the acoustics of the problem and no electromagnetic waves were involved. In any event, they did show that discharge noise in some frequency bands was either reduced or increased by sound waves penetrating the discharge at frequencies in the audio range.

The collision frequency depends, among other factors, upon gas pressure or, alternatively, neutralmolecule density. Frequently, electron density is a function of neutral density so that the plasma frequency is also pressure dependent. Thus, it seems reasonable that sound waves impinging upon a plasma should produce pressure variations that cause an alteration of the medium's complex dielectric constant. On this basis, a slightly ionized isotropic plasma has been simulated in the laboratory by a continuous $\mathrm{d}-\mathrm{c}$ discharge and disturbed by the same type of acoustic standing waves that exist in a closed cylindrical pipe driven at one end by a piston. The plasma behaves like a time-varying lossy dielectric post in a specially designed RF cavity which surrounds it, and any perturbations in $f_{p}$ and $\nu_{c}$ are seen as a modulation of the cavity output. Thus far, argon, argon-neon mixtures, and neon have been used as media in the tubes at pressures ranging from 0.1 to $30 \mathrm{~mm} \mathrm{Hg}$. However, most of the measurements have been in neon at pressures ranging from 100 to $300 \mu$.

Our initial efforts were directed toward the design and construction of compact transducers for installation into the cylindrical discharge tubes used for the production of practical laboratory plasmas. Two units were built, one a variation of a solid-dielectric electrostatic type, the other a vibrating-magneticmembrane construction for use in the low-audio range. Early experiments showed that both produced measurable perturbations in varying degrees, although the sound intensities generated by the former were insufficient for reliable measurements with a simple detection system.

\section{Transducer Design}

Among the principal considerations in the design of acoustic transducers for insertion into discharge tubes is the requirement that the unit be assembled from materials consistent with good vacuum techniques. This dictates the use of glass, ceramic and metals that can withstand the high temperatures of bake-out during discharge-tube processing, and which will not de-gas when subjected to high vacuum.

In contrast to the solid-dielectric transducer which was completely housed within the discharge tube, the moving-armature loudspeaker constructed for this apphication has most of its component parts placed outside the discharge tube and removable (fig. 1). This feature allows discharge-tube cleaning and bakeout without endangering the speaker's plastic parts and enamel-coated coil wire. The diaphragm, which is wholly inside the discharge tube, is activated through the air gap between it and the pole pieces which come through the back of the tube through a ceramic backplate. Figure 2 shows the unit's diaphragm deflection as a function of driving voltage $V_{a}$ at various acoustic frequencies $f_{a}$, made by comparing the final operating unit with static deflection measurements of its replica.

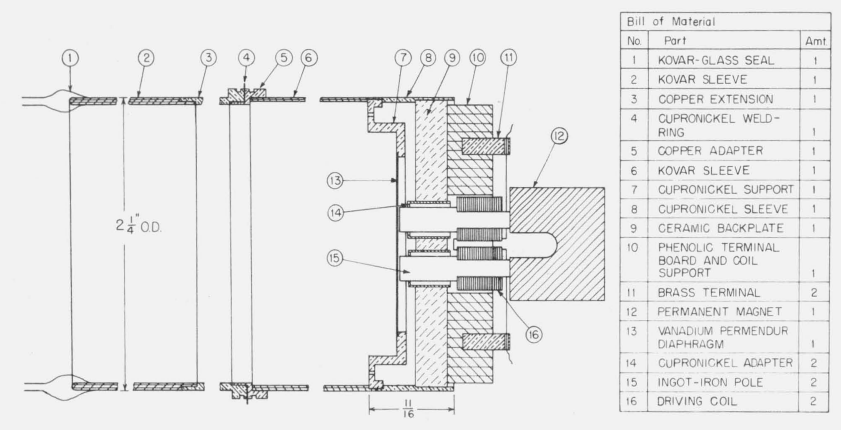

FIGURE 1. Loudspeaker based on the variation of flux in a magnetic circuit that includes a flexible membrane.

Permanent magnet, driving coils, and phenolic coil-support are external to the tube, and may be easily removed during discharge-tube processing.

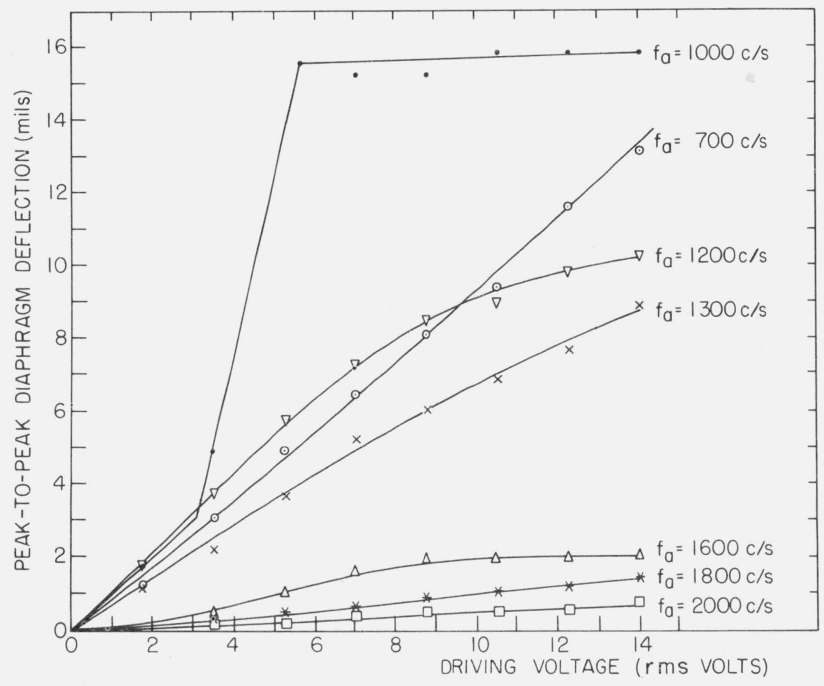

FIgURE 2. Experimentally-determined diaphragm deflections of the vibrating-magnetic-membrane loudspeaker.

These transducers have larger diaphragm deflections than the electrostatic type, but operate at much lower acoustic frequencies.

\section{Experimental Apparatus}

Figure 3 shows the 63 -mm-o.d., T-shaped, cylindrical discharge tube used to create the plasmas. A continuous d-c discharge occurs between a hot oxidecoated cathode in the T-extension and an anode whose position is adjustable by moving a soft-iron slug with an external magnet. This movable anode provides additional flexibility in varying the discharge characteristics and, in addition, serves as a variable reflector for sound waves propagated along the axis of the tube from the transducer located at the opposite end. The 


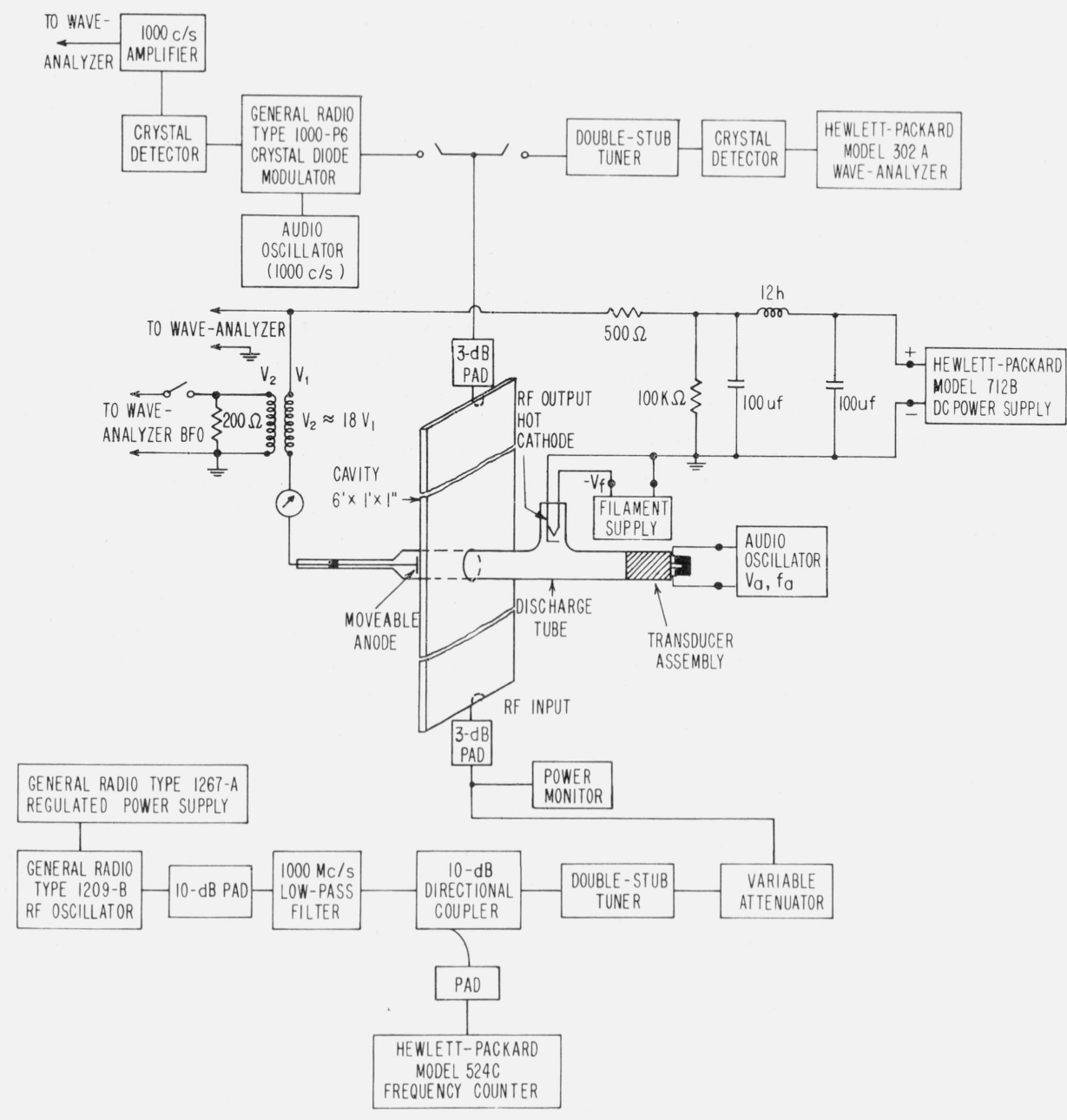

Figure 3. Physical layout and circuitry in the system for exciting and detecting acoustic waves in plasmas.

vibrating-magnetic-membrane unit is attached to the far end of the discharge tube with a large glass seal.

In the experimental setup the axis of the discharge tube is parallel to the RF electric field and perpendicular to its propagation direction in a rectangular cavity $6 \mathrm{ft}$. long with a cross section of 1 in. $\times 12$ in. The tube fits through a $25 / 8$ in. hole in the cavity center and is placed so that the cavity's narrow dimension is in the positive column of the discharge. Acoustic propagation takes place along the axis of the tube at frequencies whose wavelengths in the plasma are greater than $1 \mathrm{in}$. so that, as far as the cavity is concerned, it always sees a more-or-less uniform electron density distribution in the direction of the electric field when the plasma is perturbed.

Details of the instrumentation and circuitry in figure 3 indicate the relative simplicity of the RF detection system. Plasma and collision frequency measurements of the unperturbed medium are made using the cavity pertubation technique. In this method a modulated $\mathrm{CW}$ cavity output is used to measure the shift in the cavity resonance and change in $Q$ due to the presence of the plasma. Treating the plasma as a dielectric post creating small perturbations in the RF fields, and assuming a Bessel function radial distribution of electron density, it is possible to relate such measurements to $f_{p c}$-the plasma frequency at the discharge-tube center-and $\nu_{c}$. When the sound source is activated, the plasma-modulated cavity output is crystal detected and fed directly to a wave analyzer which has a maximum full-scale sensitivity of $30 \mu \mathrm{V}$ with $0.1-\mu \mathrm{V}$ resolution over a frequency range from $20 \mathrm{c} / \mathrm{s}$ to $50 \mathrm{kc} / \mathrm{s}$. In addition, the wave analyzer has an automatic frequency control circuit for the tracking of slowly drifting signals, and a provision to operate as a generator of very stable signals with magnitudes up to $1 \mathrm{~V}$. This latter feature is particularly useful in coupling a low-frequency signal into 
the external discharge-tube circuit while simultaneously measuring the resultant plasma modulation effect at the wave-analyzer input. BFO operation is used in this manner for calibration purposes in measuring the magnitude of plasma-frequency variations caused by the loudspeaker.

\section{Initial Measurements}

\subsection{Simultaneous Modulation of Plasma and Collision Frequencies}

Response curves similar to those shown in figure 4 indicate that the transducer modulates both the plasma and collision frequencies at the same time. This becomes clear by considering that the unperturbedcavity response for any given discharge undergoes two separate modulations when the loudspeaker is on. On one hand, the $Q$-curve (plot of reciprocal cavity insertion loss versus frequency) moves back and forth about its equilibrium position due to variations in electron density, and, if collisions were completely neglected, would produce a wave-analyzer signal whose magnitude and phase at a given electromagnetic frequency depends on the slope of the $Q$-curve. Since the wave analyzer measures only the amplitude and not the phase of a signal, a symmetrical doublehumped curve would result from RF-sweeping the cavity through its resonance, as seen from figure 5 . Maximum loudspeaker perturbation would be observed at the maximum slopes of the $Q$-curve for the electromagnetic frequencies $f_{1}$ and $f_{2}$ given by $Q \delta=Q\left(f-f_{r}\right) / f_{r}$ $= \pm \frac{1}{2} \sqrt{2}$, where $f_{r}$ is the unperturbed resonance frequency and $Q$ is the unperturbed cavity- $Q$.

If, on the other hand, the modulation of the collision frequency were the predominating effect, the wave- analyzer response as a function of the RF would be the same general shape as the $Q$-curve with maximum signal at the resonance frequency. Neglecting plasmafrequency variations, each point on the $Q$-curve can be pictured as moving up and down at the acoustic frequency as the pressure variations affect the cavity loss, which, in turn, broadens and then sharpens the $Q$-curve.

For any discharge current, equilibrium pressure, and acoustic intensity the wave-analyzer response is a superposition of the two effects, and figure 5 typifies several ways in which the respective modulations could add to give the experimental results of figure 4 . In the latter, note that the right-hand hump disappears as the current increases at constant pressure due to the fact that the collision frequency in the discharge tube also increases with current. However, for a reduced pressure the collision frequency is lower for any given discharge current so that the collision influence on the total response diminishes relative to that of the plasma-frequency variation, and the second hump is in greater evidence.

\subsection{Pressure Variations in the Discharge Tube}

Elementary acoustic theory for an adiabatic process [Morse, 1936] predicts that a piston of area $S_{p}$ vibrating at frequency $f$ with a maximum deflection $\theta_{0}$ at one end of a closed tube of length $l$ and cross-sectional area $S$ produces a maximum pressure variation $\Delta p_{0}$ at any distance $x$ from the piston given by

$$
\Delta p_{0}=\frac{\rho_{0} c^{2} \theta_{0}\left(S_{p} / S\right)}{\sin (2 \pi l / \lambda)} \frac{2 \pi}{\lambda} \cos \frac{2 \pi}{\lambda}(x-l)
$$

where $\rho_{0}$ is the equilibrium density of the medium, $c$
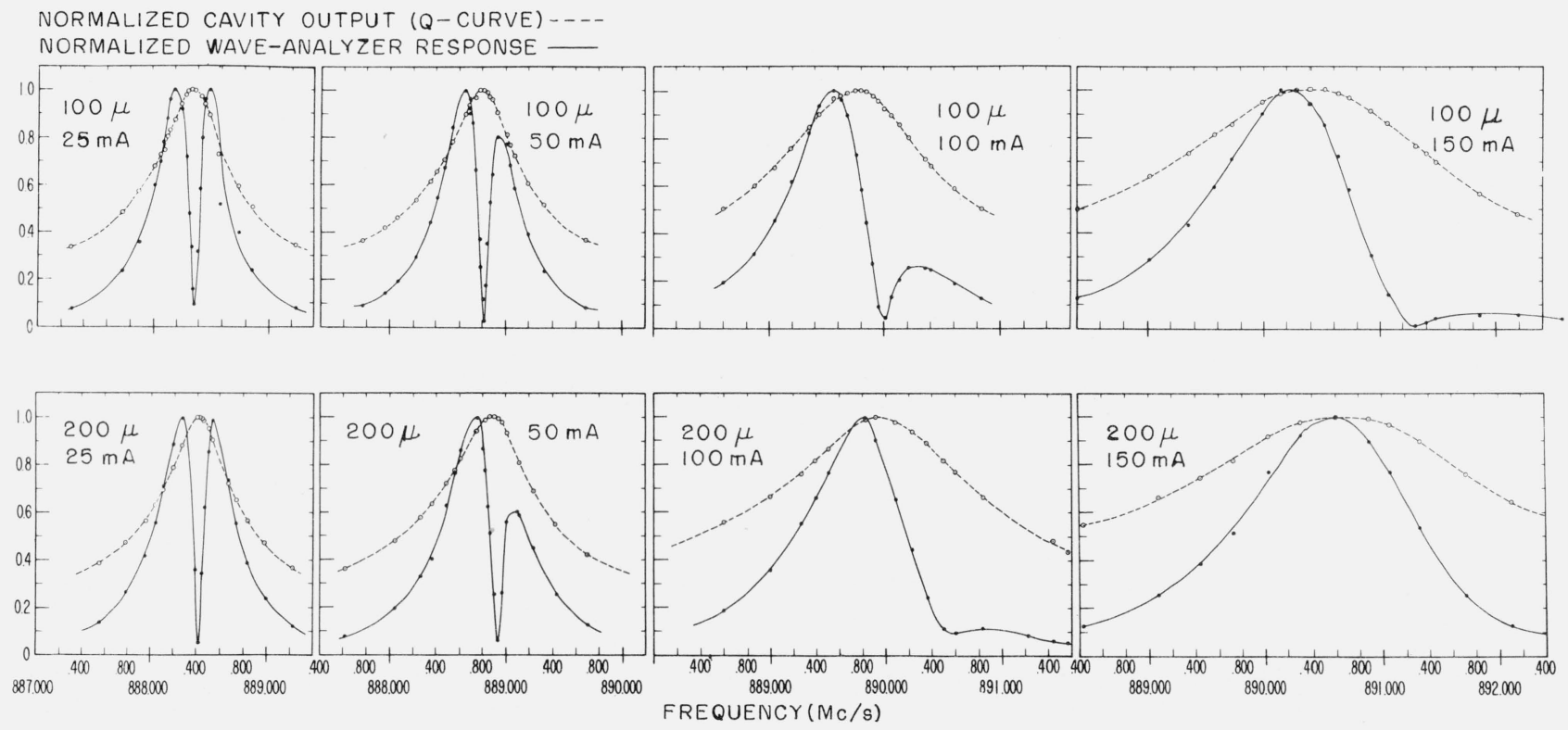

FiguRE 4. Experimental wave-analyzer response curves in perturbed neon discharges at pressures of 100 and $200 \mu$. Loudspeaker power constant $\left(V_{a}=5 \mathrm{~V} \mathrm{rms}\right)$; acoustic frequency constant at $600 \mathrm{c} / \mathrm{s}$. In each case note the relation between wave-analyzer voltage and $Q$-curve slope. 


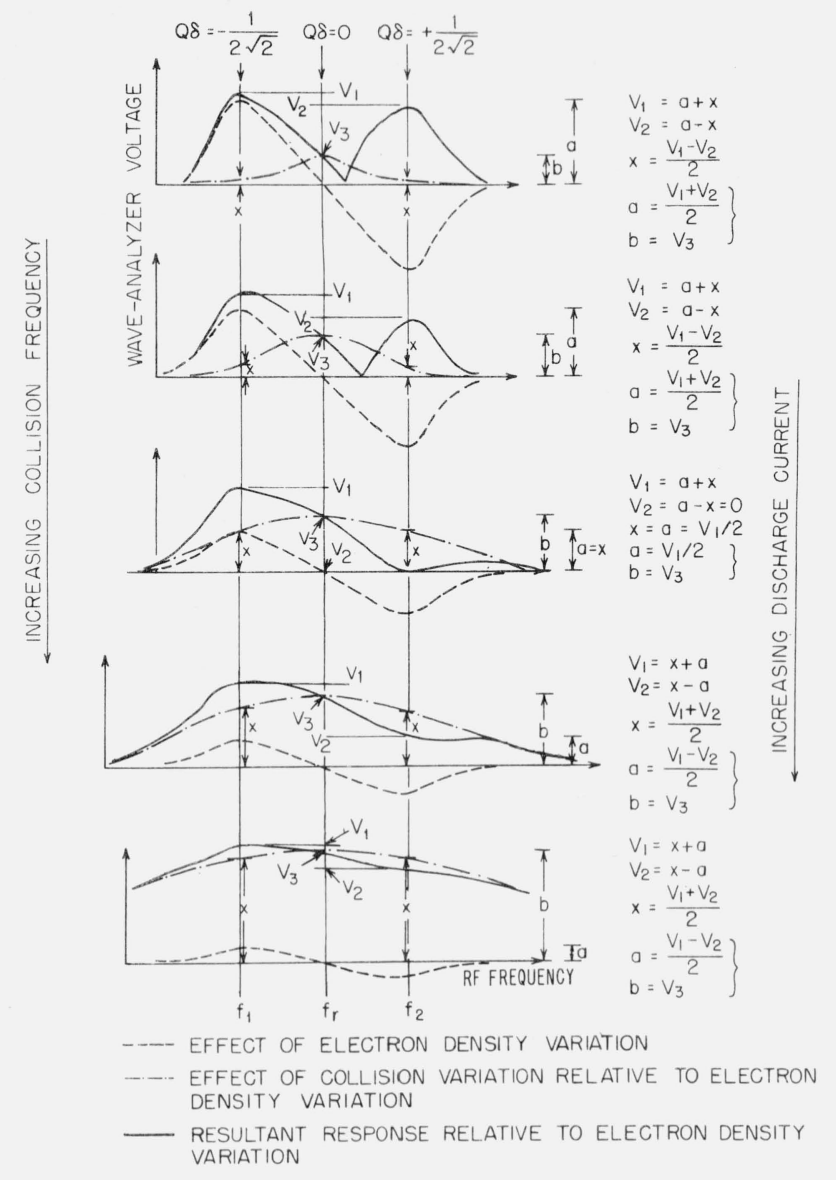

FIGURE 5. Qualitative explanation of double-humped wave-analyzer response curves showing the effect of collision frequency. By measuring $V_{1}, V_{2}$, and $V_{3}$ it is possible to determine the magnitude of variations in plasma frequency $\left(\Delta f_{p}\right)$ and collision frequency $\left(\Delta \nu_{c}\right)$ due to acoustic waves emanating from the loudspeaker. The former is proportional to $a$, while the latter is related to both $a$ and $b$.

is the speed of sound in that medium, and $\lambda=\frac{c}{f}$.

course, the actual pressure fluctuates between $p_{0}$ $+\Delta p_{0}$ (a compression) and $p_{0}-\Delta p_{0}$ (a rarefaction) at the piston frequency. Since

$$
c=\left(p_{0} \gamma / \rho_{0}\right)^{1 / 2}
$$

in an ideal gas where $\gamma$ is the ratio of specific heat at constant pressure to specific heat at constant volume,

$$
\frac{\Delta p_{0}}{p_{0}}=\gamma \frac{S_{p}}{S} \frac{2 \pi}{\lambda} \frac{\cos \frac{2 \pi}{\lambda}(x-l)}{\sin \left(\frac{2 \pi}{\lambda} l\right)} \theta_{0} .
$$

Envisioning our loudspeaker diaphragm as a moving piston propagating sound power down a closed tube with a reflector at one end acting to create a standing wave, (3) predicts that pressure variation is proportional to diaphragm deflection for a given acoustic frequency and tube geometry. If
$\Delta N_{e} / N_{e}=\Delta N / N$ ( $N_{e}$ and $N$ are electron and neutralmolecule densities)

is a valid description of the effect of such a pressure variation at any point in the ionized gas of our experiment, and since

$$
\Delta p_{0} / p_{0}=\Delta N / N,
$$

it follows that $\Delta N_{e} / N_{e}$ is proportional to $\theta_{0}$. Therefore, since $\Delta N_{e} / N_{e}=2\left(\Delta f_{p} / f_{p}\right), \Delta f_{p}$ will be proportional to the diaphragm deflection for any given discharge condition.

Using the customary assumption that $\nu_{c}$ is independent of electron velocity in discharges of certain gases (such as neon), collision frequency depends only on pressure, so that

$$
\Delta \nu_{c} / \nu_{c}=\Delta p_{0} / p_{0} .
$$

This indicates that $\Delta \nu_{c}$ should also be proportional to the diaphragm deflection for a given discharge.

Bearing in mind that the detection system is sensitive to the sum of $\Delta f_{p}$ and $\Delta \nu_{c}$, the resultant wave-analyzer responses should be proportional to diaphragm deflection if the preceding theory and assumptions are correct. Wave-analyzer responses in figure 6 show a close point-by-point correspondence to the diaphragmdeflection curves in figure 2 as a function of loudspeaker driving voltage to verify this prediction and substantiate the description given in (4) and the relationship of (6).

\subsection{Magnitude of Plasma-Frequency Variations}

The determination of the magnitudes of $\Delta f_{p}$ and $\Delta \nu_{c}$ for any given discharge and transducer operation involves the separation of the two effects in the waveanalyzer signals. A calibration scheme has been developed for this purpose which takes advantage of the double-humped responses discussed previously. Although $\Delta f_{p}$ and $\Delta \nu_{c}$ can both be found using similar procedures, our initial interest is in the former and a brief explanation of how we determine it follows.

First, the cavity-perturbation method described in section 3 is used to make a static measurement of plasma frequency at the center of the discharge $\left(f_{p c}\right)$ for two discharge currents slightly above and below the original current; then $\left(f_{p c_{2}}-f_{p c_{1}}\right) / I_{2}-I_{1}=\Delta f_{p c} / \Delta I$ $=k_{p}$ is computed (more accurate values of $k_{p}$ are found for any discharge current by differentiating the empirical relationship between $f_{p c}$ and discharge current as determined by independent measurement). A low-frequency signal from the wave-analyzer BFO is applied to the discharge tube through a transformer in the external driving circuit, and the magnitude of the resultant variation in the discharge current is measured by feeding the signal appearing across the external discharge resistor to the analyzer input (see fig. 3). This external signal modulates the entire discharge uniformly and simulates the effect of the loudspeaker in that portion of the positive column 

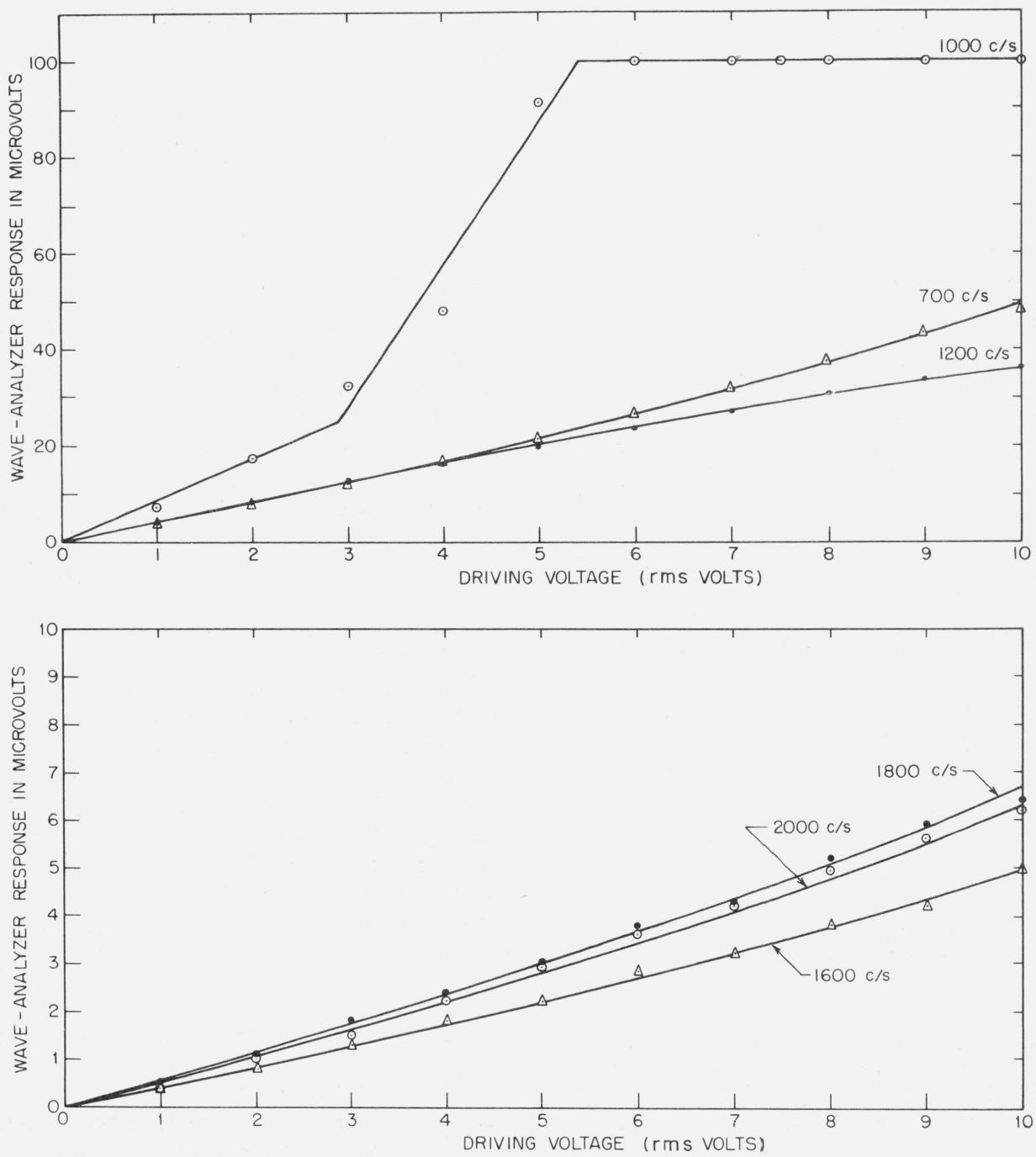

FIGURE 6. Response of a 100-ma neon discharge at $200 \mu \mathrm{Hg}$ to changes in acoustic intensity caused by variations in loudspeaker driving voltage $\mathrm{V}_{\mathrm{a}}$.

$\mathrm{RF}$ is constant $=889.963 \mathrm{Mc} / \mathrm{s}$. These data correlate with that of figure 2 to show that wave-analyzer response is proportional to diaphragm deflection.

probed by the cavity. If one were to RF-sweep through the cavity resonance, a double-humped response similar to those in figure 4 would be observed, although its right-hand hump would be noticeably lower than that of the corresponding response due to loudspeaker perturbation, probably because of a somewhat different collision process.

To a good approximation it is possible to show that $\Delta f_{p c}$ is proportional to $a$ in figure 5 , and $a$ in turn is equal to $\left(V_{1}+V_{2}\right) / 2\left[\right.$ or $\left.\left(V_{1}-V_{2}\right) / 2\right]$. Since $a$ is a maximum at the two frequencies $f_{1}$ and $f_{2}$ determined by $Q \delta= \pm 1 / 2 \sqrt{2}$, the $R F$ is adjusted to these values after a separate determination of $Q$ and resonance frequency, and wave-analyzer readings noted.

Then the BFO is disconnected and the loudspeaker turned on to a given sound power and frequency. The $\mathrm{RF}$ is again set at $f_{1}$ and $f_{2}$, the wave-analyzer tuned to the acoustic frequency involved, and the correspond- ing responses $V_{1}$ and $V_{2}$ measured (now called $V_{1}^{\prime}$ and $V_{2}^{\prime}$ respectively). Since we know that $\Delta f_{p c}$ is proportional to $V_{1}+V_{2}$ (or $V_{1}-V_{2}$ ), and that a known current variation $I_{2}-I_{1}=\Delta I$ from the wave-analyzer BFO produced a known $\Delta f_{p c}$ determined from a knowledge of $k_{p}$,

$$
\begin{aligned}
\Delta f_{p c} \text { (caused by the loudspeaker) } & =k_{p} \Delta I \frac{V_{1}^{\prime}+V_{2}^{\prime}}{V_{1}+V_{2}} \\
& \left(\text { or }=k_{p} \Delta I \frac{V_{1}^{\prime}-V_{2}^{\prime}}{V_{1}-V_{2}}\right) .
\end{aligned}
$$

Using this calibration procedure, $\Delta f_{p c}$ was measured as a function of plasma frequency for constant diaphragm deflection at several acoustic frequencies. Equation (3) predicts that $\Delta f_{p c}$ should be proportional to $f_{p c}$ at each frequency, provided that $\Delta N_{e} / N_{e}$ is equal to $\Delta N / N$. Figure 7 bears this out for low plasma fre- 


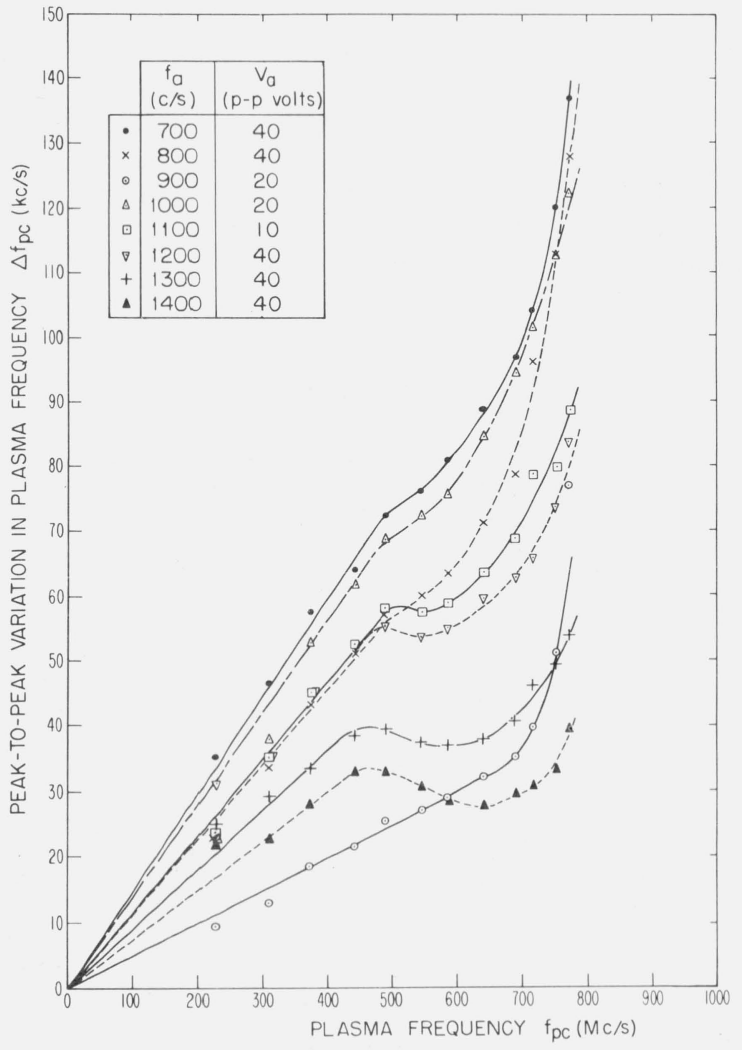

FIGURE 7. Effect of acoustic waves on the plasma frequency of a neon discharge at $200 \mu \mathrm{Hg}$.

Loudspeaker driving voltages $V_{a}$ are constant at the values indicated for each acoustic frequency $f_{a}$, and correspond to diaphragm deflections determined from figure 2 . Cavity operation is in the $\mathrm{TE}_{109}$ mode at, and near, $888 \mathrm{Mc} / \mathrm{s}$.

quencies, but each curve shows a pronounced departure from a linear relationship with "dips" at higher plasma frequencies becoming more pronounced with increases in acoustic frequency. Considering only the linear region for a moment, a comparison of $\Delta f_{p c} / f_{p c}$ determined experimentally with that predicted by simple acoustic theory shows fairly good agreement, although such correlations are not necessarily conclusive because of possible plasma side-effects and/or departures of the discharge tube construction from that assumed in the acoustic model. For example, slopes of the 700 and $1000 \mathrm{c} / \mathrm{s}$ curves in figure 7 are $1.5 \times 10^{-4}$ and $1.4 \times 10^{-4}$, respectively, corresponding to $2.1 \times 10^{-4}$ and $1.0 \times 10^{-4}$ as calculated from (3) using diaphragm deflections taken from figure 2. In (3) the equivalent piston area of the diaphragm, vibrating with maximum amplitude $\theta_{0}$ at its center, is taken as about 42 percent of the area inside the diaphragm seating surface [Miner, 1963]. ${ }^{2}$

As for the odd behavior of $\Delta f_{p c}$ at higher plasma frequencies, this is not yet resolved. Possible explanations for this observation which are presently under investigation include acoustic coupling with natural low-frequency plasma oscillations, degradation and/or

${ }^{2}$ Private communication, Bell Telephone Labs., Inc., Holmdel, N.J. enhancement of RF probing signals due to energy transfer into modulation sidebands of the original frequency [Kino and Allen, 1962], coupling of plasma column RF resonances to the avity, acoustic resonance of the discharge column itself when driven at or near critical frequencies, and the effect of the medium's ambient temperature variations on the acoustic standing-waves.

On the basis of the correlation between theoretical and experimental values of $\Delta f_{p c} / f_{p c}$ in the linear portion of the curves in figure 7 , it seems reasonable to conclude that $\Delta N_{e} / N_{e}$ does indeed equal $\Delta N / N$ for the slightly ionized gases of our discharges since we now have experimental evidence which is well explained using this relationship in two cases: constant plasma frequency, variable sound power (fig. 6); and constant sound intensity, variable plasma frequency (fig. 7). Our observation also supports the "frozen composition" assumption of Sodha and Palumbo [1964] that if the relaxation times of the ionization and deionization processes in the plasma are much greater than the period of an acoustic cycle, then the composition of the gas remains practically unchanged during such a cycle.

\section{Summary}

Measurements made thus far in this study demonstrate the feasibility of acoustically modulating both the plasma and collision frequencies of a weakly ionized gas and measuring the effect with a simple RF detection system. Although coupling to electrons is relatively inefficient using sound transducers at reduced pressures, our data indicate that pressure fluctuations of approximately $10^{-5} \mathrm{~mm} \mathrm{Hg}$ will cause plasma frequency variations in the order of $10^{-4}$ times the plasma frequency. Corresponding changes in collision frequency have not yet been determined but should follow in a straightforward way using the procedures outlined for the determination of $\Delta f_{p c}$.

Both the solid-dielectric and moving-armature types of transducer are adequate sound sources for studying the plasma modulation effect, although the latter has a greater output power in the audio range. Each design has successfully withstood the high temperatures and low pressures consistent with good vacuum-processing techniques. In addition, both units have a potential application for use as microphones in investigating sound waves or other lowfrequency oscillations generated by the plasma itself. In this case, the solid-dielectric transducer would probably be more suitable since this construction is known to have sensitivities on the order of $-90 \mathrm{~dB}$ re $1 \mathrm{~V}$ per dyne $/ \mathrm{cm}^{2}$ up to $500 \mathrm{kc} / \mathrm{s}$, many times higher than that of the moving-armature variety whose main application is as a loudspeaker. Because of their compactness and placement within the discharge tube, either type would be ideal as a probe since the discharge itself would not be disturbed.

Two immediate steps are under way in an effort to reconcile the dips that occur in the curves of figure 7 . 
One is to expand the acoustic range toward higher frequencies, the other is to make measurements in a region around a lower cavity resonance. Because there have been observations of nonlinear mixing between frequencies emanating from the loudspeaker and those present in discharges with large low-frequency oscillations, it is quite possible that the sound perturbations created by the loudspeaker couple to such plasma oscillations even when they appear to be absent from the discharge. This might be the case if the discharge were in a quasi-stable condition at plasma frequencies favoring the buildup or perhaps the onset of oscillations, especially under the influence of a substantial plasma disturbance.

With the relatively simple and controllable laboratory apparatus described here one can study the interaction between sound and longitudinal plasma waves, possible modification of the nonlinear mixing process between two RF frequencies propagating through a plasma, and the propagation of electromagnetic waves in a plasma at frequencies below the plasma frequency as predicted by Drummond [1962]. In addition, the setup might even be used to show the potential of combining sound with electromagnetic waves for plasma diagnostics. An interesting application that comes to mind is the determination of sound speedhence, neutral-particle temperature - in an inaccessible plasma, a situation one might encounter if studying the ionosphere from a remote point. This possibility could be demonstrated in the present configuration by pulsing the loudspeaker and precisely measuring incident and reflected pulses through observation of the RF modulation. However, the implementation of this experiment would require a detection system with a larger bandwidth than that now available, posing an additional problem of increased plasma-noise levels. in Sudbury, Mass. Continued encouragement and interest by R. W. P. King of Harvard University has also been a major factor in motivating the research. In addition, the author extends his sincere appreciation to Allan White, Frank Swanson, Harold Short, and Al Whitman of the Raytheon Co., Waltham, Mass. for their cooperation in fabricating the transducers and their advice on vacuum techniques.

\section{References}

Alexeff, I., and W. D. Jones (1963), Direct measurement of ionic sound-wave velocity, paper presented at the Sixth International Conference on Ionization Phenomena in Gases, Paris, France. Drummond, J. E. (1962), High frequency propagation across an ion plasma wave, Proceedings of the Fifth International Conference on Ionization Phenomena in Gases, Munich, 28 Aug.-1 Sept. 1961, ed. H. Maecker, 1, 517-526 (North-Holland Publishing Co., Amsterdam).

Goldstein, L., M. R. Roux, and J. A. Dayton, Jr. (1963), Production of acoustic waves by RF breakdown in low pressure gases, paper presented at the Sixth International Conference on Ionization Phenomena in Gases, Paris, France.

Kino, G. S., and M. A. Allen (1962), The effects of fluctuations on propagation through a plasma medium, Proceedings of the Fifth International Conference on Ionization Phenomena in Gases, Munich, 28 Aug.-1 Sept. 1961, ed. H. Maecker, 1, 602-611 (NorthHolland Publishing Co., Amsterdam).

Menzel, D. H. (1964), The general problem of ionospheric nonlinearities, paper presented at the Conference on Nonlinear Processes in the Ionosphere, Dec. 16-17, 1963. Boulder, Colo., NBS Tech. Note No. 211, 1, 1-25.

Morse, P. M. (1936), Vibration and sound, ch. VI (McGraw-Hill Book Co., Inc., New York, N.Y.).

Sessler, G. M., and M. R. Schroeder (1962), Interaction of sound waves with a gas discharge, paper presented at the Fourth International Congress on Acoustics, Copenhagen.

Sodha, M. S., and C. J. Palumbo (1964), Modulation of electromagnetic waves by acoustic waves in a plasma, Can. J. Phys. 42, $1635-1642$.

Constructive suggestions and assistance in implementing this study have been generously given by Hans J. Schmitt of the Sperry Rand Research Center 